\title{
Clear aligner: invisalign: A review
}

\author{
Roopali Sharma $^{1, *}$, Akash Rajput ${ }^{2}$, K. K. Gupta ${ }^{3}$, Himani Sharma ${ }^{4}$ \\ ${ }^{1}$ Intern, ${ }^{2}$ JRZ Omfs, ${ }^{3}$ Professor \& Head, ${ }^{4}$ Private Practice ${ }^{1}$ Dept. of Faculty of Dental Science, King George Medical, University, \\ Lucknow, Uttar Pradesh, ${ }^{2}$ Dept. of Subharti Dental College, Meerut, Uttar Pradesh, ${ }^{3}$ Dept. of Periodontology, Vyas Dental \\ College and Hospital Jodhpur, Rajasthan, ${ }^{4}$ Dept. of Periodontology, Gurugram \& Delhi, India
}

*Corresponding Author:

Email: roopalisharma1522@gmail.com

\begin{abstract}
Invisalign has given a more esthetic view to the orthodontic treatment. Clear aligners is becoming more popular among younger population not only because it provides better esthetics but better periodontal status as well during the treatment when compared to fixed orthodontic treatments. Pain and discomfort is compairtively less. Fixed orthodontic treatment has found to cause more trauma to the periodontal tissues when compared to invisalign. Clear aligners or invisalign uses 3D computer imaging technology. Invisalign serves good results in treatment of craniomandibular disorders. Prominent differences were noticed between invisalign and fixed orthodontic appliances.
\end{abstract}

Keywords: Invisalign, Clear aligner, Esthetics, Smart track.

\section{Introduction}

Pain and discomfort are common side effects of orthodontic treatment. Pain has a negative effect on patients compliance, oral hygiene, and missed appointments. Its effects on patient's daily lifes is a major reason for discontinuance of orthodontic treatment. ${ }^{1}$ Dr. H.D. Kesling introduced the concept of tooth movement using sequential removable appliance in $1945 .^{2}$ It was said" Invaslign has offered patients a variable alternative to the fixed braces and that today, practically any malocclusion can be successfully treated using, this or similar technologies." FDA approval was given for Align Technology to employ Invisalign for orthodontics use in $1998 .^{3}$

Invisalign a Removable Appliance: The Invisalign Technique uses 3D computer imaging technology to depict the complete treatment plan from the initial position to the final described position from which a series of custom-made, clear aligners are produced. Each aligner moves teeth incrementally and is worn for about weeks, then replaced by the next 1 in series until the final position is achieved. ${ }^{4}$ The aligners are removable and are made of $0.75 \mathrm{~mm}$-thick polyurethane. Each aligner is programmed to produce a precise movement on a tooth of about $0.15-0.25 \mathrm{~mm} .^{5}$ Invisalign applies intermittent forces to the teeth just as do most of the active removable appliances. ${ }^{4}$ Orthodontic treatment with Invisalign aligners could lead to root resorption as any other orthodontic treatment. ${ }^{11}$ A review by Rossini et al. demonstrated that Invisaling is effective for simple malocclusion treatment. ${ }^{11}$

\section{Materials and Methods}

Various thermoplastic materials are currently used for the fabrication of clear aligners, including polyvinyl chloride, poly urethane (PU), polyethylene texepthalate (PET), and polyethylene terepthalate glycol (PETG). ${ }^{6}$

\section{Generation of CLER Aligners}

First Generation: Earliest form of these systems were solely reliant on the aligner to achieve their results. No auxillary elements were incorporated.

Second Generation: Makes use of attachments to improve tooth movements. Clinicians could request composite buttons to be placed on the teeth and could also start to use inter maxillary elastics.

Third Generation: Attachments are now place automatically by the manufacturer's software where extrusion, de-rotation and root movements are required. Indentations in the aligners are fabricated where root to rque is needed. ${ }^{10}$

Invisalign and Fixed Appliance: A higher pain level may be observed with fixed appliances than with the removable type of appliances in general. In recent years, aligners have become one of the most growing orthodontic treatment modalities, especially invisaling. Many adult patients seek for esthetic appliances such as clear brackets, lingual appliances, and Invisalign. ${ }^{7}$ Aligers have been suggested as a viable and even preferable alternative to fixed appliances for the treatment of anterior open bites because the double thickness, of the aligners, in combination with the patient's biting force, intrudes the posterior teeth and thus aids in bite closure. ${ }^{8}$ Conventional fixed appliance results in white spot lesions, bracket induced enamel abrasion lesions. ${ }^{9}$ Traditional braces are approximately $25 \%$ more painful during the first week of treatment than invislign. ${ }^{1}$

Fixed appliances are superior in correcting occlusal contacts, posterior torque and anteroposterior discrepancies but invisalingn are similar in correcting rotations, marginal ridge heights, space closure and root alignment. $^{14}$

Aesthetics - A major Concern: Teenager patients may be particularly reluctant to undergo fixed appliance 
treatment due to drawbacks like dental and poor esthetics and for social reasons. ${ }^{12}$

Several studies reported that women under 40 preferred lingual over buccal for aesthetic and professional reasons. Similar study found a predominance of 20-30 years old females who selected Invisalign over buccal and lingual. The choice were due to esthetic consideration.

A comparison between invisalign and buccal appliances demonstrated prominent differences. Buccal patients had greater discomfort, more pain, analgesic consumption, and more functional and psychological disturbances.

According to a study compairing three orthodontic appliances (buccal, lingual and invisalign) with respect to the adult patient's perception of recovery during first 2 week after the insertion of appliance found:-

Average levels of pain were higher in the lingual and Invisalign groups, all tough the differences did not yield statistical significance. Greater consumption of analgesics in lingual patients. During first week of treatment, it was found that greater number of buccal patients that suffered from severe pain and had a high rate of consumption of analgesic compared to lingual patients. Higher oral dysfunction levels and much longer recovery time in lingual group than in both buccal and invisalign groups. The levels of impairment in oral function with invisalign were similar to buccal. Lingual patients reported more eating disturbances and a longer recovery time. The impairment associated with disturbances eating was the lowest with invisalign. More sleep disturbances were seen in lingual group. Lingual patients also had a significant longer recovery time compared to invisaling regarding difficult in swallowing, limitation in eating, and accumulation of food. $^{13}$

\section{Role and advantages of invisling:}

Invisalign is indicated in cases of mild crowding (1-6mm), mild to moderate spacing (1-6mm), nonskeletal constricted arches, and relapse after fixed appliance therapy. ${ }^{15}$ Arch expansion is possible with invisalign and may be required as a percieved need to improve the esthetics of the smile by broadening the dental arches or as a mechanism to create space for resolution of crowding. It can also be used as a way of correcting dentoalveolar posterior cross bite. ${ }^{15}$

The Invisalign system has evolved over the last 16years, and various strategies have been developed to better manage the vertical dimensions such as dental deep bite and open bite within mild to moderate range. ${ }^{16,17}$ It has been suggested that teeth moved with aligners did not undergo the typical stages of movement, as described by Krishnan and Davidovitch, because of the intermittent forces applied by the aligners. However, light continuous orthodontic forces seem to be perceived as intermittent by the periodontium, and orthodontic intermittent forces are able to produce orthodontic tooth movement with less cell damage in the periodontium. ${ }^{18}$ Treatment with invisalign provide better esthetics and comfort and lesser plaque accumulation, and is more gentle for gingival tissue than fixed orthodontic appliances ${ }^{15,19}$ It is easier to maintain oral hygiene during treatment with invisalign as toothbrushes and dental floss can access all the tooth surfaces and interproximal spaces. $^{20}$ Removability and small size of invisalign resulted in superior functional and psychological differences compared with fixed appliane, as well significantly reduced pain. ${ }^{3}$ Invisalign are also very effective for the treatment of craniomandibular disorder patients when combined with splints. $^{9}$

Disadvantages: According to a study it was shown that invisalign might not be able to produce adequate occlusal contacts as well as braces, probably because it is difficult for aligners to extrude a tooth unless there is a significant undercut and also because the aligners cover the occlusal surfaces of the teeth preventing setting of the occlusion. ${ }^{15}$ Strict requirement of patient compliance is needed for successful outcomes. ${ }^{21}$ During usage it is recommended, that aligners be removed before eating and drinking. ${ }^{6}$ The removability of invisalign is an advantage to the patient but not to clinician. $^{15}$ Above that in orthodontics, potential canditates for BPA (Bis phenol A) release include plastic materials and auxillaries such as adhesives and polycarbonate brackets and aligners. The release of BPA has been shown to be increased in alkaline environments and at high temperature, intraoral conditions might expose the aligners to transient heat shock during consumption of hot liquids ${ }^{22}$ Invisaling is limited to patient whose permanent teeth are erupted. ${ }^{15}$

The clear aligners are made of transparent thermoplastic materials which may undergo staining over time. $^{6}$

Smart Track: In 2013 Align Technology introduced the new aligner material Smart Track. The manufacturers describe the new Smart Track material as softer and more elastic than the old material. Primary goal of transitioning the Invisalign System to the Smart Track material was to optimize control of tooth movement. $^{22}$ Smart Track material is primarily composed of thermoplastic Polyurethane [PU] with some modification. Although the colour stability of the PU-based aligners is relatively less stable (particularly after coffee exposure as reported after a study), the soft PU elastomer aids in improving the elasticity and is able to produce continuous light force to the teeth, which is beneficial for orthodontic tooth movement ${ }^{6}$

\section{Conclusion}

Orthodontic treatment, in some form is now being provided by not only specialist orthodontists but general dental practitioners. $^{23}$ Overall orthodontists reported significantly more Invisalign experience than general dentists. ${ }^{24}$ Though invisalign provide better esthetics and comfort during the treatment when 
compared to fixed orthodontic treatment, it requires strict patient's compliance and have certain limitations.

\section{References}

1. David W. White, Katie C. Julien. Discomfort associated with Invisalign and traditional brackets: A randomized, prospective trial. Angle orthodontist. 2017;8.

2. Laura O. Duncan, Luis Piedade. Changes in mandibular incisor position and arch form resulting from Invisalign correction of the crowded dentition treated nonextraction. Angle Orthod.2016 Jul; 86(4): 577-83.

3. T Weir. Clear aligners in orthodontic treatmeny. Aus Dental J. 2017;62(1)1:58-62.

4. Naptitali Brezniak, Atalia Wassetein. Root Resorption Following Treatment with Aligners. Angle Ortho. 2008;78(6):110-24.

5. Jean-Philippe Houle, Luis Piedade. The prediictability of transverse changes with Invaslign. Angle Ortho. 2017;87(1):19-24.

6. Chen-Lu Liu, Wen-Tian Sun. Colour Stabilities of three types of orthodontic clear aligners exposed to staining agents. Int J Oral Sciences. 2016;8(4):246-53.

7. Koji Fujiyama, Tadashi Honjo. Analysis of pain level in cases treated with Invisalign aligner: Comparison with fixed edgewise appliance therapy. Prog Orthod. 2014;15(1):64.

8. lejandro Iglesias-Linares, Boris Sonnenberg. Orthodontically induced external apical root resorption patient's treated with fixed appliances vs removable aligners. Angle Orthod. 2017;87(1):3-10.

9. Werner SCHUPP, Julia HAUBRICH. Invislign treatment of patient's with craniomandibular disorders. Int Orthod. 2010;8:253-67.

10. Joe Hennessy and Ebratim A. Al-Awadhi. Clear aligners generations and orthodontic tooth movement. J Orthod. 2016;43(1):68-76.

11. Giulia Gay, Serena Ravera. Root resorption during orthodontic treatment with Invisalign: a radiometric study. Progress in Orthodontic. 18(1):12.

12. Jiafeng Gu, Jack Shengyu Tang. Evaluation of Invisalign treatment effectiveness and efficiency compared with conventional fixed appliances using the Peer Assessment Rating Index. Am J Orthod Dentofacial Orthop. 2017;151:259-66.

13. Miri Shalish, Rena Cooper-Kazaz. Adult patient's adjustability to orthodontic appliance. Part 1: a comparison between labial, lingual and invisalign. Eur J Orthodp. 2012;34:724-30.

14. Jiafeng Gu, Jack Shengyu Tang. Evaluation of Invisalign comapred with conventional fixed appliances using the Peer Assessment Rating Index. Am J Orthod Dentofacial Orthop. 2017;151:259-66.

15. Garret Djeu, Clarence Shelton. Outcome assessment of Invisalign and traditional orthodontic treatment compared with the American Board of Orthodontics objective grading system. Am J Orthod DEntofacial Orthop. 2005;128(3):292-8. discussion 298.

16. Roozbeh Khosravi, Bobby Cohanim. Management of overbite with the Invisalign appliance. Am J Orthod Dentofacial Orthop. 2017;151:691-99.

17. Shin K. The Insialign Appliance Could Be an Effective Modality for Treating Overbite Malocclusions Within a Mild to Moderate Range. J Evid Dent Pract. 2017;17(3):278-80.

18. Mareike Simon, Ludger Keilig. Treatment outcome and efficacy of an aligner technique- regarding incisor torque, premolar derotation and molar distabilization. BMC Oral Healt. 14:68.

19. A.Azaripour, J.Weusmann, B. Mahmood. Braces versus Invisalign: gingival parameters nd patient's satisfaction during treatment: a cross sectional study. BMC Oral Health. 2015;15:69.

20. Rainer-Reginald Miethke, Klaudia Brauner. A Comparison of thr Periodontal Health of Patient's during treatment with the Invisalign System and with Fixed Lingual Appliances. J Orofac Orthop. 2007;68:223-31.

21. Anne-Kathrin Braschher, Dietmar Zuran. Patient suervey on Invisalign treatment Comparing the Smart Track Material to the previous aligner material. J Orofac Orthop. 2017;13.

22. Madahar Arun, Qureshi, Usman and Ama Johal. Orthodontic treatment Modalities: a qualitative assessment of Internet Information. J Orthod. 2017;44(2):82-89. 